\section{An example with interesting controllability and stabilisation properties}

Ron M. Hirschorn* Andrew D. Lewis ${ }^{\dagger}$

$25 / 02 / 2005$

\section{Abstract}

A simple three-state system with two inputs is considered. The system's controllability is determined using properties of vector-valued quadratic forms. The quadratic structure is then used as the basis for the design of a homogeneous, discontinuous, stabilising feedback controller. The paper should be seen as an attempt to relate controllability of a system from a point to stabilisability of the system to the same point.

Keywords. controllability, stabilisation

AMS Subject Classifications. 93B05, 93D15

\section{Introduction}

As is well-known, for any system for which it is possible to reach an equilibrium point with open-loop controls from any nearby point, i.e., for systems that are locally asymptotically controllable, there exists a continuous control Lyapunov function [Sontag 1983, Sontag and Sussmann 1995]. The matter of whether it is possible to stabilise a system by feedback is then answerable if one can use a continuous Lyapunov function to design stabilising feedback, as can be done fairly easily in the case of a differentiable control Lyapunov function [Sontag 1989]. That this is indeed possible has been proved by Clarke, Ledyaev, Sontag, and Subotin [1997], using ideas from nonsmooth analysis. Of course, the biggest practical obstruction to the utility of these results is the determination of a control Lyapunov function for a given example, or class of examples. Indeed, a reasonably fair description of much of the present stabilisation literature would be that it consists of the pursuit of classes of systems for which control Lyapunov functions can be found. Many of these classes of systems possess control Lyapunov functions that are differentiable in a (possibly punctured) neighbourhood of the equilibrium point, and so, by the theorem of Artstein [1983], are stabilisable a feedback control law that is $C^{\infty}$, except possibly at the equilibrium point

For systems that are only stabilisable by discontinuous state feedback, less work has been done along the lines of determining control Lyapunov functions. In such cases, Artstein's Theorem ensures that the control-Lyapunov function will not be differentiable. In this paper we initiate a discussion on the relationship between controllability and stabilisability. *Professor, Department of Mathematics and Statistics, Queen's University, Kingston, ON K7L 3N6, CANADA

Email: ron@mast.queensu.ca, URL: http://www.mast.queensu.ca/ ${ }^{\sim}$ ron/

†Associate Professor, Department of Mathematics and Statistics, Queen's University, Kingston, ON K7L 3N6, CANADA

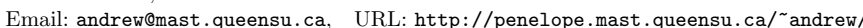

As is well-known, controllability from a point does allow on to conclude stabilisability using smooth feedback to the same point (see the discussion in [Bacciotti 1992], for example). However, there does not appear to be a systematic exploration of the relationship between controllability from a point and stabilisation, possibly discontinuous, to the same point. Indeed, the matter of connecting the geometric theory of controllability (e.g., using Lie brackets, etc.) with the analytical theory of stabilisation (e.g., using control Lyapunov functions) seems to be an important one that has not been touched upon in a significant way. It is in this direction that we aim, in this paper, to make a small first step by using the controllability properties of a system as a basis for the design of a feedback control law.

Briefly, in Section 2 we consider a class of systems that are locally controllable from a given point. While it ought to be possible for this class of systems to describe a general procedure for designing a discontinuous stabilising feedback, this appears to be somewhat nontrivial. Therefore, in this preliminary paper we content ourselves with perhaps the simplest example in this class. Even for this example, as can be seen, the state feedback is quite complex.

\section{A class of controllable systems}

In this section we consider a class of systems that is controllable at "second-order." The systems we consider fall into the larger class considered by Hirschorn and Lewis [2002]. First we provide the details of the class of systems, then we indicate a general idea, using homogeneity, for beginning the design of a stabilising state feedback. This will put us in position, in Section 3, to design a stabilising feedback for one of the systems from the class.

2.1. The systems and their controllability. We consider a system whose state space is $\mathbb{R}^{n}$, and we partition $\mathbb{R}^{n}$ as $\mathbb{R}^{m} \times \mathbb{R}^{n-m}$. The states we then denote by $(\boldsymbol{x}, \boldsymbol{y}) \in \mathbb{R}^{n} \times \mathbb{R}^{n-m}$. The governing equations we consider are

$$
\begin{aligned}
\dot{\boldsymbol{x}}(t) & =\boldsymbol{u}(t) \\
\dot{\boldsymbol{y}}(t) & =\boldsymbol{Q}(\boldsymbol{x}(t)),
\end{aligned}
$$

where $\boldsymbol{u} \in \mathbb{R}^{m}$ is the control, and $\boldsymbol{Q}: \mathbb{R}^{m} \rightarrow \mathbb{R}^{n-m}$ is a quadratic map, i.e., $\boldsymbol{Q}(\lambda \boldsymbol{x})=\lambda^{2} \boldsymbol{Q}(\boldsymbol{x})$ for all $\boldsymbol{x} \in \mathbb{R}^{m}$ and $\lambda \in \mathbb{R}$. This class of system is clearly not linearly controllable. However, the following result describes the nonlinear controllability properties of the system, and we refer the reader to [Hirschorn and Lewis 2002] for further details (see also [Basto-Gonçalves 1998]). We denote by $\operatorname{conv}(S)$ denotes the convex hull of a set $S$, and by int $(S)$ the interior of $S$.

2.1 THEOREM: Suppose that the controls for the system (2.1) take values in a subset $U \subset \mathbb{R}^{m}$ for which $\mathbf{0} \in \operatorname{int}(\operatorname{conv}(U))$. Then the system (2.1) is small-time locally controllable from $(\mathbf{0}, \mathbf{0})$ if and only if $\mathbf{0} \in \operatorname{int}(\operatorname{conv}(\operatorname{image}(\boldsymbol{Q})))$.

Based on this theorem on the controllability of the system (2.1), it seems reasonable to assume that $\mathbf{0} \in \operatorname{int}(\operatorname{conv}(\operatorname{image}(\boldsymbol{Q})))$ in order to ensure that $(\mathbf{0 , 0})$ is stabilisable using state feedback. Indeed, if $\mathbf{0} \notin \operatorname{int}(\operatorname{conv}(\operatorname{image}(\boldsymbol{Q})))$, then it is easy to see that the system is not asymptotically controllable to $(\mathbf{0 , 0})$, and so therefore not stabilisable by feedback of any sort. 
2.2. A general construction using homogeneity. In this section we outline some general initial steps that reduce the stabilisation problem for the system (2.1) first to a sphere, then to the product of a sphere with a disk. The initial assumption is that the state feedback u: $\mathbb{R}^{m} \times \mathbb{R}^{n-m} \rightarrow \mathbb{R}^{m}$ has the property that the clos under the action of $\mathbb{R}$ on $\mathbb{R}^{m} \times \mathbb{R}^{n-m}$ given by $\Phi(s,(\boldsymbol{x}, \boldsymbol{y})) \mapsto\left(\mathrm{e}^{s} \boldsymbol{x}, \mathrm{e}^{2 s} \boldsymbol{y}\right)$. This means, in language perhaps more familiar to control theoreticians, that we ask that the closed-loop system be homogeneous with respect to some dilation. We refer the reader to [Kawski 1995] for a discussion of various notions of homogeneity, in particular, the group invariance version we use here. In any case, since we are assuming the closed-loop system to be invariant under the action $\Phi$, its flow will drop to a flow on the set of orbits of the group action, i.e., on the quotient space. Omitting the trivial orbit which is the origin, the orbits are generally parabolic curves in $\mathbb{R}^{m} \times \mathbb{R}^{n-m}$ of the form $\mathbb{R}_{+} \ni t \mapsto\left(t \boldsymbol{x}, t^{2} \boldsymbol{y}\right)$. In the case of a point where $\boldsymbol{y}=\mathbf{0}$, the orbit is then a line. In either case, again omitting the trivial orbit at the origin, each orbit intersects an appropriate sphere in exactly one place, so the set of orbits is parameterised by an $(n-1)$-sphere. It is convenient to realise this parameterisation explicitly by using a very particular submanifold of $\mathbb{R}^{m} \times \mathbb{R}^{n-m}$ that is homeomorphic to a sphere, but is not the standard Euclidean sphere. The sphere we want is defined by the following homogeneous norm: $\rho: \mathbb{R}^{m} \times \mathbb{R}^{n-m} \rightarrow \mathbb{R}:(\boldsymbol{x}, \boldsymbol{y}) \mapsto \sqrt{\|\boldsymbol{x}\|^{2}+\|\boldsymbol{y}\|}$, where $\|\cdot\|$ is the standard Euclidean norm. The function $\rho$ is distinguished by the fact that it is an invariant function for the group action $\Phi$. Thus, for example, level sets of $\rho$ are mapped to other level sets of $\rho$ under the group action. For this reason, there is a 1-1-correspondence between group orbits and points in the level set $\rho^{-1}(1)$. We shall use the set $\rho^{-1}(1)$ as an explicit model for the set of orbits for the group action (again, omitting the orbit $(\mathbf{0}, \mathbf{0}))$. The canonical projection onto the quotient is then given by $\pi: \mathbb{R}^{m} \times \mathbb{R}^{n-m} \rightarrow \rho^{-1}(1):\left(\rho(\boldsymbol{x}, \boldsymbol{y})^{-1} \boldsymbol{x}, \rho(\boldsymbol{x}, \boldsymbol{y})^{-2} \boldsymbol{y}\right)$

Now, again assuming that the state feedback $\boldsymbol{u}$ is invariant under the group action $\Phi$, the closed-loop vector field will be $\pi$-related to a vector field on $\rho^{-1}(1)$, the model for the quotient. Moreover, the vector field on $\rho^{-1}(1)$ is defined by $(\boldsymbol{x}, \boldsymbol{y}) \mapsto T_{(\boldsymbol{x}, \boldsymbol{y})} \pi(\boldsymbol{u}, \boldsymbol{Q}(\boldsymbol{x}))$, for $(\boldsymbol{x}, \boldsymbol{y}) \in \rho^{-1}(1)$. A straightforward computation then gives the governing equations on $\rho^{-1}(1)$ as

$$
\begin{aligned}
& \dot{\boldsymbol{x}}=-\frac{1}{2} \boldsymbol{x} \hat{\boldsymbol{y}}^{T} \boldsymbol{Q}(\boldsymbol{x})+\left(\boldsymbol{I}_{m}-\boldsymbol{x} \boldsymbol{x}^{T}\right) \boldsymbol{u}, \\
& \dot{\boldsymbol{y}}=\left(\boldsymbol{I}_{n-m}-\boldsymbol{y} \hat{\boldsymbol{y}}^{T}\right) \boldsymbol{Q}(\boldsymbol{x})-2 \boldsymbol{y} \boldsymbol{x}^{T} \boldsymbol{u},
\end{aligned}
$$

where $\hat{\boldsymbol{y}}$ is the unit vector, with respect to the standard norm in $\mathbb{R}^{n-m}$, in the direction of $y$

A further simplification can be achieved by considering in more detail the geometry of $\rho^{-1}(1)$. Note that this is not a submanifold of $\mathbb{R}^{m} \times \mathbb{R}^{n-m}$ since it is not smooth at points where $\boldsymbol{y}=\mathbf{0}$. Therefore, the closed-loop vector field can be expected to have some poor behaviour on the sphere

$$
S=\left\{(\boldsymbol{x}, \boldsymbol{y}) \in \rho^{-1}(1) \mid \boldsymbol{y}=\mathbf{0}\right\} .
$$

Indeed, as we will see with our explicit feedback law in Section 3, the differential equations governing the trajectories will change when they pass through the set $S$. This motivates removing the set $S$ from the analysis, and treating it as a sort of boundary region for the system. What then is the geometry of $\rho^{-1}(1) \backslash S$ ? If one thinks about it for a moment, the variables $\boldsymbol{x} \in \rho^{-1}(1) \backslash S$ take values in the unit disk

$$
\mathbb{D}^{m}(\mathbf{0})=\left\{\boldsymbol{x} \in \mathbb{R}^{m} \mid\|\boldsymbol{x}\|=1\right\} .
$$

Moreover, for each point $\boldsymbol{x} \in \mathbb{D}^{m}(\mathbf{0})$, there is associated a sphere's worth of points $\boldsymbol{y} \in \mathbb{R}^{n-m}$ with the property that $(\boldsymbol{x}, \boldsymbol{y}) \in \rho^{-1}(1)$. The radius of this sphere goes from a maximum 1 when $\boldsymbol{x}=\mathbf{0}$, and decreases to 0 as $\boldsymbol{x}$ approaches the boundary of $\mathbb{D}^{m}(\mathbf{0})$. It is convenient, therefore, to normalise $\boldsymbol{y}$ to have unit radius to give an explicit diffeomorphism of $\rho^{-1}(1) \backslash S$ therefore, to normalise $\boldsymbol{y}$ to have unit radius to give an explicit diffeomorphism of $\rho^{-1}(1) \backslash S$
with $\mathbb{D}^{m}(\mathbf{0}) \times \mathbb{S}^{n-m-1}$. This is accomplished with the diffeomorphism $(\boldsymbol{x}, \boldsymbol{y}) \mapsto\left(\boldsymbol{x}, \hat{\boldsymbol{y}}=\frac{\boldsymbol{y}}{\|\boldsymbol{y}\|}\right)$. Applying this change of coordinates to the equations (2.2), we get the differential equations

$$
\begin{aligned}
& \dot{\boldsymbol{x}}=-\frac{1}{2} \boldsymbol{x} \hat{\boldsymbol{y}}^{T} \boldsymbol{Q}(\boldsymbol{x})+\left(\boldsymbol{I}_{m}-\boldsymbol{x} \boldsymbol{x}^{T}\right) \boldsymbol{u}, \\
& \dot{\hat{\boldsymbol{y}}}=\left(\boldsymbol{I}_{n-m}-\hat{\boldsymbol{y}} \hat{\boldsymbol{y}}^{T}\right) \frac{\boldsymbol{Q}(\boldsymbol{x})}{1-\|\boldsymbol{x}\|^{2}},
\end{aligned}
$$

which are valid for $(\boldsymbol{x}, \hat{\boldsymbol{y}}) \in \mathbb{D}^{m}(\mathbf{0}) \times \mathbb{S}^{n-m-1}$

It is equations (2.3) that will form the starting point for our analysis of the example in the next section.

\section{A stabilising feedback law for a simple example}

In this section we consider a simple example from the general class of systems represented by equation (2.1). After presenting the system, we reduce it to the homogeneous sphere. Then we present a control strategy based on our observations about the nature of the reduced control system.

3.1. The system and its reduction to the homogeneous sphere. The example we consider in this section is the special case of the system $(2.1)$, where $n=3, m=2$, and $Q\left(x_{1}, x_{2}\right)=x_{1}^{2}-x_{2}^{2}$. The resulting differential equations are

$$
\dot{x}_{1}=u_{1}, \quad \dot{x}_{2}=u_{2}, \quad \dot{y}=x_{1}^{2}-x_{2}^{2}
$$

It is easy to verify that $0 \in \operatorname{int}(\operatorname{conv}(\operatorname{image}(Q)))$, so the system (3.1) is small-time locally controllable from $((0,0), 0)$ by Theorem 2.1. This is, in some sense, the simplest example of a controllable system of the form (2.1).

In terms of the reduction of the system using homogeneity to the equations (2.3), we note that, since $n-m-1=0$, the second of equations (2.3) is trivial $\left(\mathbb{S}^{n-m-1}=\{-1,1\}\right.$ ). Therefore, we only need reproduce the first of equations (2.3), and these are determined to be

$$
\begin{aligned}
& \dot{x}_{1}=\mp \frac{1}{2} x_{1}\left(x_{1}^{2}-x_{2}^{2}\right)+\left(1-x_{1}^{2}\right) u_{1}-x_{1} x_{2} u_{2}, \\
& \dot{x}_{2}=\mp \frac{1}{2} x_{2}\left(x_{1}^{2}-x_{2}^{2}\right)-x_{1} x_{2} u_{1}+\left(1-x_{2}^{2}\right) u_{2},
\end{aligned}
$$

which should be thought of as a system on two copies, indexed by \pm 1 , of the unit disk $\mathbb{D}^{2}(0,0)$ in the $\left(x_{1}, x_{2}\right)$-plane. The " $\mp$ " in the first term on the right-hand side corresponds to taking $\hat{y}=+1$ (this gives the minus sign) or $\hat{y}=-1$ (this gives the plus sign).

Let us make some observations about the system (3.2). 


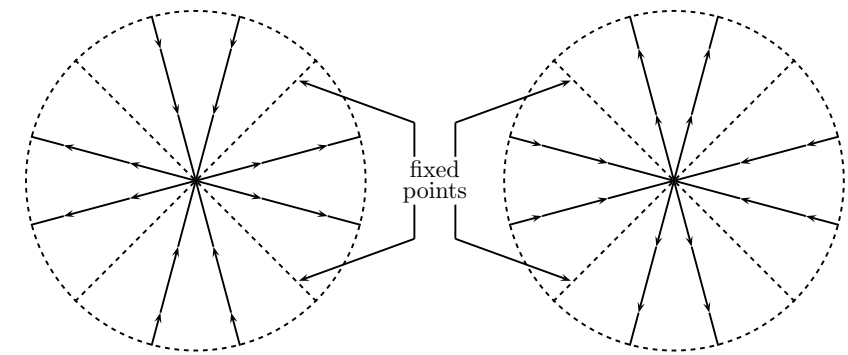

FiguRE 1. Integral curves for the drift vector field on $\mathbb{D}^{2}(0,0)$ when $\hat{y}=-1$ (left) and when $\hat{y}=1$ (right)

1. At a point $\left(\left(x_{1}, x_{2}\right), y\right) \in \mathbb{D}^{2}(0,0) \times\{-1,1\}$, the drift vector field points in the direction $\pm\left(x_{1}, x_{2}\right)$, i.e., either away from or towards the origin, depending on the sign of $x_{1}^{2}-x_{2}^{2}$ and the sign of $y$. In Figure 1 we show the character of the drift vector field for the cases $\hat{y}=-1$ and $\hat{y}=1$.

2. The controls appear in the two equations like so:

$$
\left[\begin{array}{cc}
1-x_{1}^{2} & -x_{1} x_{2} \\
-x_{1} x_{2} & 1-x_{2}^{2}
\end{array}\right]\left[\begin{array}{l}
u_{1} \\
u_{2}
\end{array}\right]
$$

The coefficient matrix has nonzero determinant in the interior of $\mathbb{D}^{2}(0,0)$, but the determinant vanishes as $\left(x_{1}, x_{2}\right)$ approaches the boundary of $\mathbb{D}^{2}(0,0)$. Thus the control directions are arbitrarily specifiable in the interior of $\mathbb{D}^{2}(0,0)$.

3. On the boundary of $\mathbb{D}^{2}(0,0)$, the control vector field becomes tangent to the boundary Thus, at the boundary of $\mathbb{D}^{2}(0,0)$, one can only control the tangential component of the flow, not the normal component.

Note that all of these observations are also valid for the general equations (2.3).

3.2. The stabilisation strategy. The objective of our stabilisation strategy is to shape the closed-loop flow on the reduced space in such a way that it guarantees stability for the unreduced flow. This idea is based on general discussions along these lines by Kawski [1991]. To undertand our strategy, suppose that we have a state feelbak $\boldsymbol{u} \mathbb{D}^{m}(\mathbf{0}) \times \mathbb{S}^{n-m-1}$ $\mathbb{R}^{m}$ for the reduced equations $(2.3)$ on the homogeneous sphere. This then gives a state $\mathbb{R}^{m}$ for the reduced equations $(2.3)$ on the homogeneous
feedback $\overline{\boldsymbol{u}}: \mathbb{R}^{m} \times \mathbb{R}^{n-m} \rightarrow \mathbb{R}^{m}$ for the full system $(2.1)$ by

\section{$\bar{u}(\boldsymbol{x}, \boldsymbol{y})=\boldsymbol{u}(\pi(\boldsymbol{x}, \boldsymbol{y}))$}

The resulting closed-loop system on $\mathbb{R}^{m} \times \mathbb{R}^{n-m}$ is then, of course, invariant with respect to the group action $\Phi$. Therefore, equilibria for the reduced system on $\mathbb{D}^{m}(\mathbf{0}) \times \mathbb{S}^{n-m-1}$ correspond to group orbits that are also trajectories for the closed-loop system. Therefore it is of importance to describe the trajectories in $\mathbb{R}^{m} \times \mathbb{R}^{n-m}$ corresponding to equilibria on $\mathbb{D}^{m}(\mathbf{0}) \times \mathbb{S}^{n-m-1}$

The key to this is the following general result.
3.1 Proposition: As in the preceding discussion, let $\boldsymbol{u}: \mathbb{D}^{m}(\mathbf{0}) \times \mathbb{S}^{n-m-1} \rightarrow \mathbb{R}^{m}$ be a state feedback for the reduced equations (2.3) on the homogeneous sphere, and let $\overline{\boldsymbol{u}}: \mathbb{R}^{m} \times$ $\mathbb{R}^{n-m} \rightarrow \mathbb{R}^{m}$ be the corresponding state feedback for the system (2.1). Let $\left(\boldsymbol{x}_{0}, \hat{\boldsymbol{y}}_{0}\right) \in$ $\mathbb{D}^{m}(\mathbf{0}) \times \mathbb{S}^{n-m-1}$ be an equilibrium point for the reduced closed-loop system and let $\left(\boldsymbol{x}_{0}, \boldsymbol{y}_{0}\right)$ be the associated point in $\rho^{-1}(1)$. Then the following statements hold:

(i) the corresponding closed-loop trajectory through $\left(\boldsymbol{x}_{0}, \boldsymbol{y}_{0}\right)$ in the full state space $\mathbb{R}^{m} \times$ $\mathbb{R}^{n-m}$ is given by $t \mapsto\left(\mathrm{e}^{\alpha t} \boldsymbol{x}_{0}, \mathrm{e}^{2 \alpha t} \boldsymbol{y}_{0}\right)$, where $2 \alpha \boldsymbol{y}_{0}=\boldsymbol{Q}\left(\boldsymbol{x}_{0}\right)$;

(ii) the corresponding closed-loop trajectory through $\left(\boldsymbol{x}_{0}, \boldsymbol{y}_{0}\right)$ in the full state space $\mathbb{R}^{m} \times$ $\mathbb{R}^{n-m}$ tends to $(\mathbf{0 , 0})$ if and only if $\boldsymbol{y}_{0}^{T} \boldsymbol{Q}\left(\boldsymbol{x}_{0}\right)<0$.

Proof: Let $t \mapsto(\boldsymbol{x}(t), \boldsymbol{y}(t))$ be the closed-loop trajectory for the system (2.1). Since this is also the image of a one-parameter subgroup of $\mathbb{R}$ under the action $\Phi$, we have $\boldsymbol{x}(t)=\mathrm{e}^{\alpha t} \boldsymbol{x}_{0}$ and $\boldsymbol{y}(t)=\mathrm{e}^{2 \alpha t} \boldsymbol{y}_{0}$ for some $\alpha \in \mathbb{R}$. Since $\dot{\boldsymbol{y}}(t)=\boldsymbol{Q}(\boldsymbol{x}(t))$, the first part of the result easily follows. The second part follows from multiplying the expression $2 \alpha \boldsymbol{y}_{0}=\boldsymbol{Q}\left(\boldsymbol{x}_{0}\right)$ on the left by $\boldsymbol{y}_{0}^{T}$.

The upshot of this result, when applied to our system (3.1), is that we should design the closed-loop system on $\mathbb{D}^{2}(0,0) \times\{-1,1\}$ to only have equilibrium points in the regions in Figure 1 where the integral curves for the reduced drift vector field are directed away from the origin. More precisely, this suggests the following scheme for designing a stabilising control law via the design of a state feedback for the reduced system.

3.2 Problem: Find a state feedback $\boldsymbol{u}: \mathbb{D}^{2}(0,0) \times\{-1,1\} \rightarrow \mathbb{R}^{2}$ with the following properties.

(i) The closed-loop system on $\mathbb{D}^{2}(0,0) \times\{-1,1\}$ has a finite number of equilibrium points, all occurring in a region where the integral curves of the reduced drift vector field are directed away from $(0,0)$.

(ii) The $\omega$-limit set of every point in $\mathbb{D}^{2}(0,0) \times\{-1,1\}$ consists of one of the finite number of equilibrium points. That is to say, all trajectories tend to one of the equilibrium points which correspond, in the full system (3.1), to trajectories which asymptotically approach $((0,0), 0)$.

To obtain a solution to this problem, we employ a strategy as follows.

1. We break the homogeneous sphere $\mathbb{D}^{2}(0,0) \times\{-1,1\}$ into four regions as shown in Figure 2.

2. In each region we ask that there be two equilibrium points located and with stability as in Figure 2.

3. In each of the four regions, we ask that the dynamics be the gradient flow of a potential function designed to have a minimum at the stable equilibrium, a maximum at the unstable equilibrium, and to have boundary behaviour consistent with the fact that we can only modify the tangential component of the closed-loop flow on boundary of $\mathbb{D}^{2}(0,0) \times\{-1,1\}$.

Of course, the difficult step is the last one, and indeed it is not a priori clear that it is possible. However, it is possible, and one can easily check that the potential function

$$
V_{3}\left(x_{1}, x_{2}\right)=3\left(-2+x_{1}^{2}-5 x_{2}\right)\left(-1+x_{1}^{2}+x_{2}^{2}\right)^{2}+\frac{1}{4}\left(x_{1}^{2}-x_{2}^{2}\right)\left(-5+3 x_{1}^{2}+3 x_{2}^{2}\right)
$$



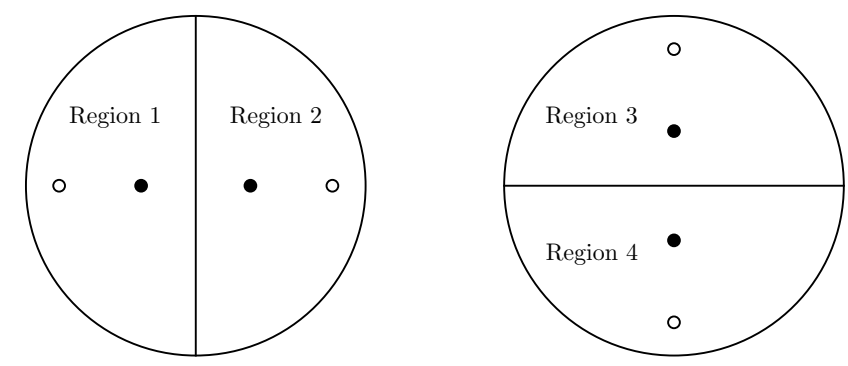

FIgURE 2. The location of equilibria on $\mathbb{D}^{2}(0,0) \times\{-1,1\}$. A $\circ$ means an unstable equilibrium and a $\bullet$ means a stable equi-
librium. The left figure corresponds to $\hat{y}=-1$ and the right figure corresponds to $\hat{y}=1$.

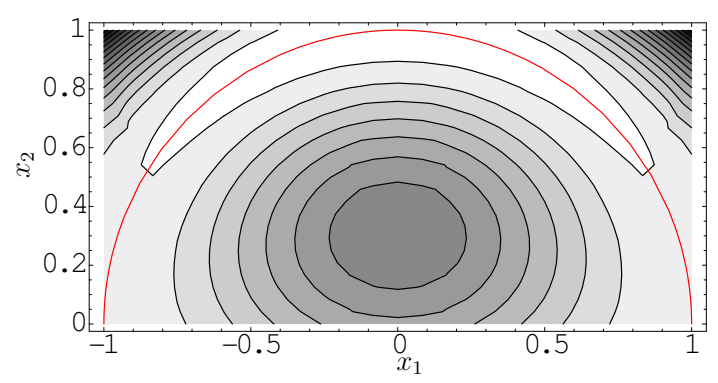

Figure 3. A contour plot for $V_{3}$. Darker regions correspond to smaller values of the function.

has the desired properties in Region 3 in Figure 2. We show in Figure 3 a contour plot for $V_{3}$ so the reader can understand the character of the function. One then defines the potential function in Regions 1, 2, and 3 by

$$
V_{1}\left(x_{1}, x_{2}\right)=V_{3}\left(-x_{2}, x_{2}\right), \quad V_{2}\left(x_{1}, x_{2}\right)=V_{3}\left(x_{2},-x_{1}\right), \quad V_{4}\left(x_{1}, x_{2}\right)=V_{3}\left(x_{1},-x_{2}\right),
$$

respectively. The resulting potential function defined on all of $\mathbb{D}^{2}(0,0) \times\{-1,1\}$ we denote by $V$. Note that to obtain the controls, one simply sets the right-hand side of equation (3.2) equal to $-\operatorname{grad} V$, and then solves for the control. Because $V$ is designed to have the appropriate boundary behaviour, this procedure is well-defined. Of course, the function $V$ is not differentiable. However, at points of discontinuity of the derivative, one can make a choice of one of the two possible limiting values for $\operatorname{grad} V$.

In Figure 4 we show the closed-loop phase portrait on $\mathbb{D}^{2}(0,0) \times\{-1,1\}$. Note that the unstable equilibria indicated in Figure 2 are very close to the boundary of the disk, and so
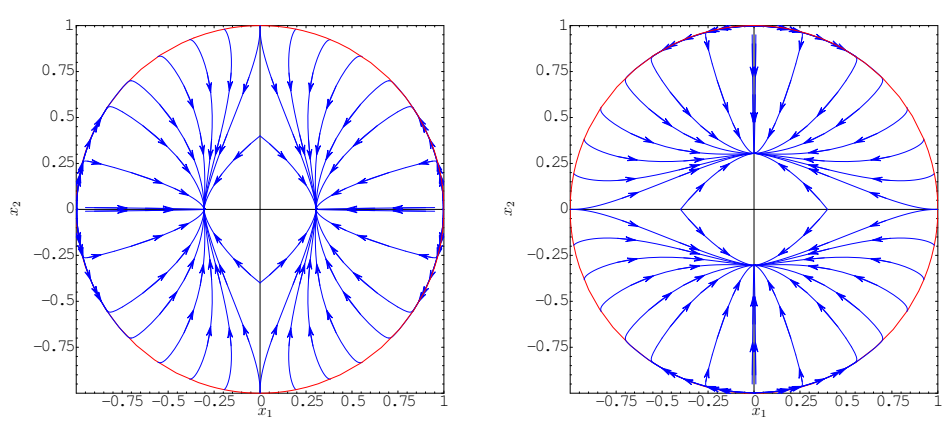

Figure 4. The closed-loop phase portrait for $\hat{y}=-1$ (left) and $\hat{y}=1$ (right)

are hard to see in Figure 4.

One can glue the two copies of the disk from $\mathbb{D}^{2}(0,0) \times\{-1,1\}$ along their boundary to get a vector field on a manifold homeomorphic to the two-sphere. This vector field will have discontinuities on the boundary where the gluing takes place, and also on the boundaries between Regions 1 and 2, and between Regions 3 and 4. One needs to be careful about the manner in which one defines solutions to these discontinuous vector fields. For example, if one goes with the Filippov notion of a solution, there will be unwanted equilibria at the origins of the disks. The resolution of this is to instead make an arbitrary choice for the value of the vector field along the lines of discontinuity. One can then define solutions in the sense of Carathéodory, and the difficulties of unwanted Filippov equilibria will be avoided.

In Figure 5 we show the obligatory simulation where the states decay to zero. Note that the performance is not all that good, but this can be improved by changing the potential function. In particular, it turns out that to achieve faster rates of exponential decay along the homogeneous rays, one should move the stable fixed points in Figure 2 closer to the boundary. Also, performance can be improved by making the potential well deeper at the stable equilibria. However, these are design issues that are somewhat peripheral to what we are trying to achieve here, which is to use the controllability structure as the basis for the design of a stabilising feedback.

\section{Extensions and future work}

The immediate modest objective of the work we describe here is to design a stabilising feedback for a general system of the type described by (2.1). Certain of the ideas used in Section 3 to motivate our control strategy are also applicable to completely general systems of the type of (2.1). However, the three-dimensional system we consider is special in at least two ways. 

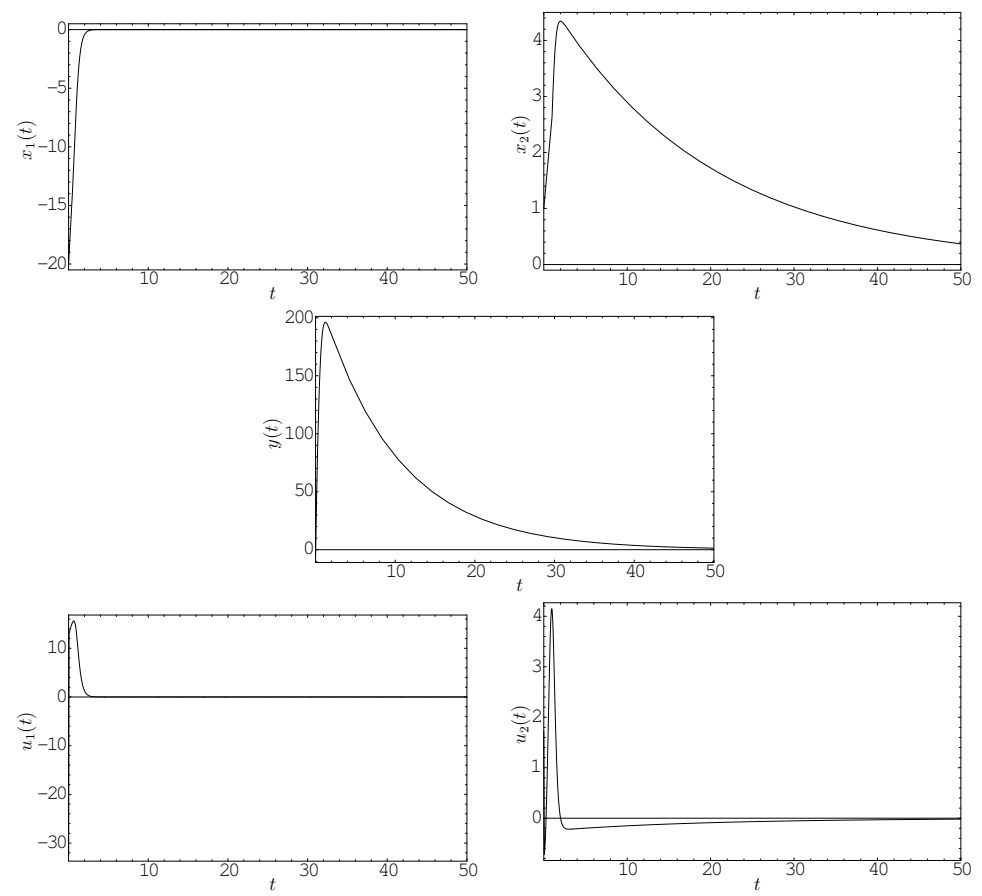

FIGURE 5. Simulation showing states and controls for an initial condition of $(1,1,1)$

1. The reduced dynamics of (2.3) have no $\boldsymbol{y}$-component for the example we consider. This makes the matter of designing the feedback on the homogeneous sphere significantly simpler than will be the case in general.

2. The quadratic function $Q$ is surjective for our example. This is key because it allows us, for every point $\boldsymbol{y} \in \mathbb{R}^{n-m}$, to find an $\boldsymbol{x}$ such that $\boldsymbol{Q}(\boldsymbol{x})=-\lambda \boldsymbol{y}$ for $\lambda>0$. This is an essential part of our control strategy. An example of a system for which $\boldsymbol{Q}$ is not surjective is

$$
\begin{gathered}
\dot{x}_{1}=u_{1}, \quad \dot{x}_{2}=u_{2}, \quad \dot{x}_{3}=u_{3}, \\
\dot{y}_{1}=x_{1} x_{2}, \quad \dot{y}_{2}=x_{1} x_{3}, \quad \dot{y}_{3}=x_{2} x_{3} .
\end{gathered}
$$

For this system, it only holds for certain $\boldsymbol{y}$ 's that we can find an $\boldsymbol{x}$ such that $\boldsymbol{Q}(\boldsymbol{x})=-\lambda \boldsymbol{y}$ for $\lambda>0$. This complicates the picture significantly.
Despite these two difficulties, it seems reasonable that one can generalise the control strategy we present here. In so doing, one will arrive at explicit feedback stabilisers for a class of systems which have interesting controllability properties.

And, as we mentioned in the introduction, one of the ideas of interest to us is to investigate the relationship between controllability from a point and stabilisability to a point. To this end, it would be useful to use the methodology we describe here to determine a control Lyapunov function for the system. The potential function on $\mathbb{D}^{2}(0,0) \times\{-1,1\}$ gives rise to a continuous, but not differentiable, control Lyapunov function on the homogeneous sphere $\rho^{-1}(1)$. However, this does not extend to a control Lyapunov function for the full system (3.1). However, perhaps it is possible to arrive at a corresponding control Lyapunov function, using the geometric controllability structure of the system.

In any case, there certainly appears to be much progress to be made in understanding the relationship between controllability and stabilisation.

\section{References}

Artstein, Z. [1983] Stabilization with relaxed controls, Nonlinear Analysis. Theory, Methods, and Applications, 7(11), 1163-1173.

Bacciotti, A. [1992] Local Stabilizability of Nonlinear Control Systems, number 8 in Series on Advances in Mathematics for Applied Sciences, World Scientific, Singapore, New Jersey, London, Hong Kong, ISBN 981-02-0713-1.

Basto-Gonçalves, J. [1998] Second-order conditions for local controllability, Systems \& Control Letters, 35(5), 287-290.

Clarke, F. H., Ledyaev, Y. S., Sontag, E. D., and Subotin, A. I. [1997] Asymptotic controllability implies feedback stabilization, Institute of Electrical and Electronics Engineers. Transactions on Automatic Control, 42(10), 1394-1407.

Hirschorn, R. M. and Lewis, A. D. [2002] Geometric local controllability: Second-order conditions, in Proceedings of the 41st IEEE Conference on Decision and Control, pages 368-369. Institute of Electrical and Electronics Engineers, Las Vegas, NV.

Kawski, M. [1991] Families of dilations and asymptotic stability, in Analysis of Controlled Dynamical Systems (Lyon, 1990), B. Bonnard, B. Bride, J.-P. Gauthier, and I. Kupka, editors, pages 285-294, number 8 in Progress in Systems and Control Theory, Birkhäuser, Boston/Basel/Stuttgart, ISBN 0-8176-3576-9.

- [1995] Geometric homogeneity and applications to stabilization, in Nonlinear Control Systems Design, A. Krener and D. Mayne, editors, pages 147-152, Elsevier Publishing Company, Amsterdam/London/New York.

Sontag, E. D. [1983] A Lyapunov-like characterization of asymptotic controllability, SIAM Journal on Control and Optimization, 21(3), 462-471.

- [1989] A "universal" construction of Artstein's theorem on nonlinear stabilization, Systems \& Control Letters, 13(2), 117-123. 
AN EXAMPLE WITH INTERESTING CONTROLLABILITY AND STABILISATION PROPERTIES11

Sontag, E. D. and Sussmann, H. J. [1995] Nonsmooth control-Lyapunov functions, in Proceedings of the 34th IEEE Conference on Decision and Control, pages 2799-2805, Institute of Electrical and Electronics Engineers, New Orleans, LA. 